\title{
Individualized Case-Control Study of Accelerated Partial Breast Irradiation by Multicatheter Intertstitial Brachytherapy in Japanese Patients with Breast Cancer
}

\author{
Kazuhiko Sato ${ }^{1}$, Yoshio Mizuno ${ }^{1}$, Hiromi Fuchikami ${ }^{1}$, Masahiro Kato ${ }^{2}$, Takahiro Shimo ${ }^{2}$, \\ Jun Kubota ${ }^{2}$, Naoko Takeda ${ }^{1,3}$, Yuko Inoue ${ }^{3}$, Hiroshi Seto ${ }^{4} \&$ Tomohiko Okawa $^{5}$ \\ ${ }^{1}$ Department of Breast Oncology, Tokyo-West Tokushukai Hospital, Tokyo, Japan \\ ${ }^{2}$ Department of Radiation Oncology, Tokyo-West Tokushukai Hospital, Tokyo, Japan \\ ${ }^{3}$ Inoue Ladies Clinic, Tokyo, Japan \\ ${ }^{4}$ Seto Hospital, Saitama, Japan \\ ${ }^{5}$ Health Evaluation Center, Utsunomiya Memorial Hospital, Tochigi, Japan \\ Correspondence: Kazuhiko Sato, Department of Breast Oncology, Tokyo-West Tokushukai Hospital, 3-1-1 \\ Matsubara, Akishima, Tokyo 196-0003, Japan. E-mail: kazsato.boc@gmail.com
}

Received: March 24, 2013 Accepted: April 14, 2013 Online Published: April 23, 2013

doi:10.5539/cco.v2n1p127 URL: http://dx.doi.org/10.5539/cco.v2n1p127

This study was not funded by any public or private grant.

All authors declare no conflict of interest.

\begin{abstract}
Abbreviations
BCS, breast-conserving surgery; WBI, whole-breast irradiation; APBI, accelerated partial breast irradiation; ICC, individualized case-control; CT, computed tomography; MRI, magnetic resonance imaging; IBTR, ipsilateral breast tumor recurrence.
\end{abstract}

\begin{abstract}
Background: Breast-conserving surgery (BCS) followed by whole-breast irradiation (WBI) are the standard of care for treating early-stage breast cancer. The efficacy of accelerated partial breast irradiation (APBI) has recently been investigated as an alternative to WBI. Although we previously reported that multicatheter brachytherapy in APBI is feasible in Japanese patients, long-term data of several APBI techniques have not yet been obtained. Here we estimated the long-term efficacy of multicatheter brachytherapy in Japanese patients with breast cancer to validate its use an individualized case-control (ICC) analysis. Method: From October 2008 to March 2013, a total of 184 consequent patients with 188 tumors underwent BCS followed by multicatheter brachytherapy. A total of 120 consecutive patients (mean age, 55.1 years) with 122 tumors (pN0) who had at least 1 year of follow-up were enrolled in this analysis. Before lumpectomy, the insertion of applicators and delivery doses were simulated by computed tomography. After confirming the free margins and negative sentinel node metastases by frozen section analysis, the applicators were inserted. APBI therapy was initiated on the same day of surgery on the basis of the dose distribution analysis, at 32 Gy in 8 fractions over 5-6 days with a 2 -cm tumor margin coverage. Regarding our prospective follow-up policy in this observational study, all patients had a predefined schedule including clinical examination every 3-4 months and mammography and breast magnetic resonance imaging (MRI) every 12 months. We performed ICC analysis to estimate the number of patients with ipsilateral breast tumor recurrence (IBTR). The 10-year risk of IBTR for each patient when undergoing with WBI was calculated using the web-based decision-making tool IBTR! and was adjusted during the real-time follow-up period using data obtained from an overview by the Early Breast Cancer Trialists' Collaborative Group. Results: The median follow-up period was 3.1 years (range, 1.1-4.4 years). Most tumors (92.6\%) were $\leq 2 \mathrm{~cm}$ in diameter, and $89.4 \%$ were estrogen receptor-positive. Hormone therapy was administered in $86.7 \%$ and adjuvant chemotherapy in $20.0 \%$ patients. If all patients received WBI after BCS, we estimated
\end{abstract}


IBTR in this group to be 1.1-2.8. Although 1 IBTR was observed, no tumor bed recurrence was recorded. Conclusions: Although this study involved only a small number of patients and short follow-up period, only 1 IBTR case was recorded, which was within the estimated range of local recurrence. Multicatheter brachytherapy using this technique may offer the same rates of local control as WBI in Japanese patients.

Keywords: individualized case-control analysis, breast cancer, accelerated partial breast irradiation, multicatheter brachytherapy

\section{Introduction}

Breast-conserving surgery (BCS) followed by whole-breast irradiation (WBI) is reportedly as effective as mastectomy and has is now the standard of care for treating early-stage breast cancer (Fisher et al., 2002; Veronesi et al., 2002). Although the aims of radiation to the entire breast are to decrease the risk of local recurrence and enhance survival rates (Vinh-Hung, \& Verschraegen, 2004; Clarke et al., 2005; Early Breast Cancer Trialists' Collaborative Group, 2011), severe and long-term adverse events such as rib fracture, lung injury, and late cardiovascular toxicity occasionally occur because at least 5-6 weeks of daily visits to radiation institutions are required. Despite the potential advantages, $15 \%-30 \%$ patients who undergo BCS refuse WBI (Farrow, Hunt, \& Samet, 1992; Lazovich et al., 1991; Ballard-Barbash et al., 1996; Athas et al., 2000; Schroen et al., 2005).

Current evidence reveals that no subset of patients should spare radiation therapy after BCS (Fisher et al., 2002; Fyles et al., 2004; Hughes et al., 2004), and local recurrence after BCS with WBI is most likely to occur in the vicinity of the lumpectomy site (Fowble et al., 1990; Gage et al., 1995; Smith et al., 2000; Krauss et al., 2004). Therefore, the efficacy and feasibility of accelerated partial breast irradiation (APBI) as an alternative to WBI in patients with early-stage breast cancer have been evaluated in many phase II and III studies (Polga'r et al., 2002; Polga'r et al., 2004; Veronesi et al., 2010; Vaidya et al., 2010; Polgár et al., 2007).

In October 2008, we initiated a prospective observational study on APBI with multicatheter brachytherapy as an alternative to WBI after BCS. Four-year follow-up data have been reported showing a few instances of local recurrence and a low level of adverse events (Sato et al., 2012). However, this observational study was based on a retrospective chart review; therefore, it is difficult to validate the procedure because of the small number of included patients, varying clinical backgrounds, and a short follow-up period. In this study, we estimated the long-term efficacy of our technique in Japanese patients with breast cancer using individualized case-control (ICC) analysis (Vaidya et al., 2011).

\section{Patients and Methods}

\subsection{Patients and Study Enrolment}

Patients with early-stage and node-negative breast cancer were studied by prospective observation to evaluate the efficacy of APBI with multicatheter brachytherapy. A detailed summary and eligibility criteria have been reported previously (Sato et al., 2012). Our criteria included patient age $\geq 40$ years, histologically documented breast cancer, no prior treatment, unifocal disease, maximum tumor diameter $\leq 3.0 \mathrm{~cm}$, and confirmation of negative margins and sentinel node metastasis using frozen section analysis. Individual indications for this technique and postoperative care were at the discretion of the attending physicians. All patients provided written informed consent, and this registry study was approved by the institutional review board of our hospital.

A total of 184 consecutive patients with 188 tumors treated by multicatheter brachytherapy from October 2008 to March 2013 were enrolled in a registry trial designed to collect data. This ICC analysis focused on node-negative patients with follow-up longer than 1 year. From the above, 124 consecutive patients with 126 treated tumors became the subjects of this analysis. Two patients who had received WBI [1 each for previous breast cancer treatment and disqualifying pathology (positive margins)] were excluded. Two patients were regarded as being lost to follow-up because of attendance at other hospitals (no recurrence had been reported). These 4 patients were excluded from the analysis. Therefore, 120 patients with 122 tumors were subjected to ICC analysis (Figure 1).

In the follow-up of 188 breast tumors receiving multicatheter APBI after BCS, 122 tumors were subjected to ICC analysis. 
Enrollment

Breast tumors received multicatheter APBI

$$
(\mathrm{n}=188)
$$

Follow-up

Analysis

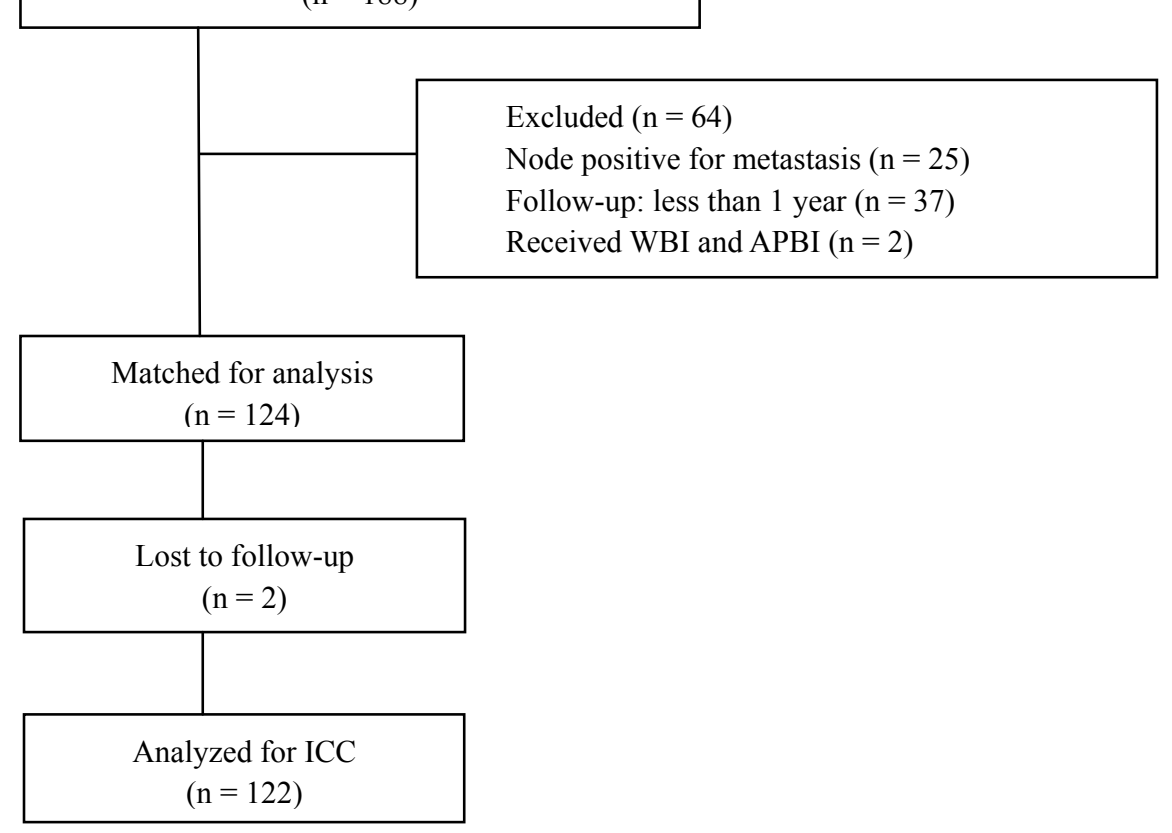

Figure 1. CONSORT diagram

\subsection{Treatment Schema}

BCS followed by APBI with multicatheter brachytherapy has previously been reported (Sato et al., 2012; Sato et al., 2011). The procedure involved the insertion of applicators and delivery doses simulated by preoperative computed tomography (CT). Lumpectomy and sentinel node biopsy were performed, and then the surgical margins and SNs were confirmed as negative by specimen mammography and frozen section analysis. Applicators for the introduction of iridium (Ir) wires were inserted according to the preoperative CT-based simulation.

The lumpectomy cavity was identified on postoperative CT scans with the help of hemoclips. The planned target volume was defined as the estimated tumor volume plus a $20-\mathrm{mm}$ margin. Dose distribution analysis was performed on the basis of postoperative CT using dose-volume histograms. Patients received high-dose rate brachytherapy with a Ir-192 source following the irradiation plan on the Nucletron PLATO system (Version UPS: 11.3; Nucletron Trading BV, Veenendaal, The Netherlands). APBI was initiated on the day of primary surgery, at a dose of 32 Gy in 8 fractions over 5-6 days. Fractions delivered twice daily were separated by an interval of at least $6 \mathrm{~h}$.

\subsection{Assessment of Outcomes}

The prospective follow-up policy was designed so that all patients had a predefined schedule including clinical examination every 3-4 months and annual mammography. Contrast-enhanced breast magnetic resonance imaging (MRI) was performed every 12 months for the first 5 years of follow-up to detect ipsilateral breast tumor recurrence (IBTR) and regional nodal failure as early as possible.

For analysis, IBTR was classified by clinical location in relation to the initial lumpectomy cavity. Tumor bed recurrence (true recurrence or marginal miss) was defined as recurrence of the treated cancer within or immediately adjacent to the primary tumor site. Failure elsewhere was defined as IBTR several centimeters from the primary site and was generally considered to be a new primary cancer (Recht et al., 1988; Giess et al., 1999; Beitsch et al., 2012).

Any postoperative complications noted at follow-up were documented using the National Cancer Institute Common Terminology Criteria for Adverse Events (CTCAE) version 3.0 (http://ctep.cancer.gov/protocolDevelopment/electronic_applications/docs/ctcaev3.pdf) to evaluate acute and late toxicities: radiation dermatitis, wound infection, skin breakdown, and fat necrosis requiring multiple aspiration. 
Acute toxicity was defined as toxicity occurring within 90 days of the first day of APBI (Ott et al., 2007).

\subsection{Individual Case-Control Analysis}

The method used to validate the effect of our brachytherapy technique on tumor control after BCS was introduced as follows: First, the estimated number of IBTRs in this cohort over 10 years was calculated by the summation of all risks predicted using the web-based IBTR! program (Sanghani et al., 2010), which calculates the 10-year risk of IBTR with and without the addition of WBI after BCS. Second, the estimated risk of IBTR was adjusted by the actual duration of the follow-up period using the Early Breast Cancer Trialists' Collaborative Group data (Clarke et al., 2005). Third, the estimated number of IBTRs in patients receiving BCS with or without WBI was compared with the actual number. This method was introduced as "individual case-control analysis" (Vaidya et al., 2011).

The mathematical model predicted the 10-year IBTR rate for individual patients by input of tumor characteristics such as age, tumor diameter, grade, surgical margins, lymphovascular invasion, and status of systemic therapy (Clarke et al., 2005). When the extent of surgical margins was not specifically addressed in the details, reportedly well-defined negative and suspicious margins were regarded as those $>2 \mathrm{~mm}$ and $0-2 \mathrm{~mm}$, respectively. Patients with bilateral breast cancer at presentation were included in the database twice to calculate IBTR rates.

\section{Results}

\subsection{Patient Demographics and Tumor Characteristics}

In the new cohort of 120 patients with 122 tumors, the median follow-up was 3.1 years (range, 1.1-4.4), and patient demographics (mean age, 55.1 years) are listed in Table 1.

Table 1. Patient demographics $(n=120)$ and tumor characteristics $(n=122)$

\begin{tabular}{|c|c|c|}
\hline Characteristics & $\mathrm{N}$ & $\%$ \\
\hline Mean age (range, years) & $55.1(30-92)$ & \\
\hline$<40$ years & 8 & 6.7 \\
\hline $40-69$ years & 101 & 84.2 \\
\hline$>70$ years & 11 & 9.1 \\
\hline \multicolumn{3}{|l|}{ Pathological tumor stage } \\
\hline pTis & 14 & 11.5 \\
\hline pT1 & 99 & 81.1 \\
\hline pT2 & 9 & 7.4 \\
\hline \multicolumn{3}{|l|}{ Grading (nuclear grade) } \\
\hline Grade I & 85 & 69.7 \\
\hline Grade II & 19 & 15.6 \\
\hline Grade III & 1 & 0.8 \\
\hline NA & 17 & 13.9 \\
\hline \multicolumn{3}{|l|}{ Margins at first excision } \\
\hline Free & 109 & 89.4 \\
\hline Suspicious & 7 & 5.7 \\
\hline DCIS only & 6 & 4.9 \\
\hline \multicolumn{3}{|c|}{ Hormone receptor status $(>10 \%)$} \\
\hline $\mathrm{ER}^{+}$or $\mathrm{PgR}^{+}$ & 109 & 89.4 \\
\hline $\mathrm{ER}^{-}$and $\mathrm{Pg} \mathrm{R}^{-}$ & 13 & 10.6 \\
\hline \multicolumn{3}{|l|}{ Her2/neu status } \\
\hline$+\left(\mathrm{IHC}^{+}\right.$or $\left.\mathrm{FISH}^{+}\right)$ & 14 & 11.5 \\
\hline - (IHC 3- and $\left.\mathrm{FISH}^{-}\right)$ & 98 & 80.3 \\
\hline NA & 10 & 8.2 \\
\hline
\end{tabular}

Abbreviations: DCIS, ductal carcinoma in situ; ER, estrogen receptor; PgR, progesterone receptor. Her2/neu, human epidermal growth factor receptor 2; IHC, immunohistochemistry; FISH, fluorescence in situ hybridization. 
The majority of tumors $(92.6 \%)$ were $\leq 2 \mathrm{~cm}$ in diameter, whereas $89.4 \%$ were hormone receptor-positive. Nine lesions (7.4\%) required excisional biopsy for a definitive diagnosis. A total of 109 patients $(90.8 \%)$ underwent adjuvant systemic therapy; of these, $104(86.7 \%)$ received hormonal therapy, $24(20.0 \%)$ received chemotherapy, and $9(7.5 \%)$ received adjuvant trastuzumab.

\subsection{Acute and Late Toxicities}

All acute and late toxicities were generally mild, with none Grade 3 or 4 , and no patient required secondary surgery because of toxicity. Table 2 presents an analysis of variables associated with Grade 0 vs. Grade 1/2 skin toxicity. The distribution of patients according to late skin toxicity score at final follow-up was also analyzed. There were no cases of Grade 3/4 skin toxicity, but 2 lesions (1.6\%) developed seroma requiring multiple aspiration of fat necrosis and 7 (5.7\%) developed wound infection followed by wound breakdown.

Table 2. Acute and chronic toxicities after treatment $(\mathrm{n}=122)$

\begin{tabular}{lll}
\hline Acute Toxicity & $\mathrm{N}$ & $\%$ \\
\hline $\begin{array}{l}\text { Hemorrhage } \\
\text { Grade 1 }\end{array}$ & 2 & 1.6 \\
$\quad$ Wound infection & 1 & 0.8 \\
$\quad$ Grade 2 & & \\
$\quad$ Radiation dermatitis & 15 & 12.3 \\
$\quad$ Grade 1 & 1 & 0.8 \\
$\quad$ Grade 2 & 7 & 5.7 \\
$\quad$ Wound infection with wound breakdown & & 1.6 \\
\hline Chronic Toxicity & 2 & \\
Seroma requiring multiple aspirations or fat necrosis & 2 & \\
\hline
\end{tabular}

\subsection{Validation of Tumor Control by Multicatheter Brachytherapy}

The calculated total of 10-year IBTR with and without WBI was 4.1 and 13.7, respectively. The actual follow-up period varied between 1 and 5 years. According to Early Breast Cancer Trialists' Collaborative Group data, $27.0 \%$ IBTRs occurring within 10 years in patients with WBI may occur 1 year after surgery and $67.0 \%$ may occur at 5 years. When patients did not receive WBI, 30.8\% and 78.4\% IBTRs may occur at 1 and 5 years, respectively. Therefore, if all patients received WBI after BCS, 1.1-2.8 IBTRs may occur, whereas if no radiation therapy was administered after BCS, 4.2-10.8 IBTRs would occur.

On the other hand, at final follow-up, 1 patient died from a non-original breast cancer-related cause with no local recurrence, and local failure occurred in 3 patients, including 1 IBTR (failure elsewhere) and 2 lymph node recurrences with no distant metastasis. Therefore, the actual number of IBTRs in this cohort was in the range of that expected in patients receiving WBI.

\section{Discussion}

Among various APBI techniques introduced as an alternative to WBI, multicatheter brachytherapy is the best established technique and mostly frequently evaluated. However, compared with studies in European countries and the United States, few studies have evaluated this technique in Asian patients with breast cancer (Mitsumori, \& Hiraoka, 2008; Yeo et al., 2010). To our knowledge, this is one of the largest prospective observational studies from Asia, demonstrating the feasibility of BCT using multicatheter brachytherapy and showing acceptable clinical outcomes.

The efficacy and safety of APBI as part of BCT have been investigated since the late 1990s, and various APBI techniques including brachytherapy, hypofractionated conformal radiation therapy, and intraoperative linear acceleration or electron therapy have been evaluated at many institutions (Njeh, Saunders, \& Langton, 2010). Although each technique has its potential benefits and limitations and limited data have been currently reported from large randomized clinical trials (McCormick, 2005), the use of brachytherapy has significantly increased since the FDA approval of the balloon-based brachytherapy device (Grace et al., 2010; Barton, 2011). A substantial proportion of potential candidate patients in Japan are interested in the convenience of APBI as an alternative to WBI. In fact, most patients in this study lived at a distance from our institution, which discouraged 
frequent visits for WBI; furthermore, the estimated direct costs of multicatheter brachytherapy, covered by national insurance in Japan, are substantially less than those of conventional WBI, which might favor its more wide spread use (Grobmyer et al., 2013). Moreover, in this technique, wide-field partial breast irradiation with safe margins in the skin and chest wall can be achieved, even in patients with relatively small breasts, by appropriate arrangement of the number and lines of catheters inserted. According to a recent large case-control study, incidental exposure of the heart to radiotherapy increased the rate of coronary events in patients with and without preexistence cardiac risk factors (Darby et al., 2013). In our multicatheter brachytherapy, the radiation dose to the heart is almost none.

However, we await the results of a National Surgical Adjuvant Breast and Bowel Project B39/Radiation Therapy Oncology Group 0413 Phase III trial; meanwhile, Early Breast Cancer Trialists' Collaborative Group data on the relationship between effects on local control and breast cancer mortality show that 1 death from breast cancer could be avoided for every 4 local recurrences prevented (Clarke et al., 2005; Early Breast Cancer Trialists' Collaborative Group, 2011). Moreover, a significantly increased risk of subsequent mastectomy and higher rates of short-term wound and skin complications were reported in patients receiving brachytherapy than in those receiving WBI (Presley et al., 2012; Grace et al., 2012). Those results were obtained from a Medicare claim-based study on relatively elderly patients, and there were limitations with regard to retrospective analyses and detailed information on subsequent complications and reasons for mastectomy.

It may be simple to demonstrate the safety of the APBI technique in 1 single institution by comparing reported adverse event rates. In fact, all toxicities related to radiation therapy were mild in this study. On the other hand, the evaluation of efficacy with regard to local control is much more difficult because the risk of recurrence after $\mathrm{BCT}$ has been reported to be associated with a variety of unfavorable factors such as young patients, relatively large tumors, pure ductal carcinoma in situ, and positive or suspicious margins. The types of systemic treatment also varied. Tumor subtype was recently claimed to be a factor relevant to local recurrence (Millar et al., 2009; Gabos et al., 2010; Hattangadi-Gluth et al., 2012). Because of the low level of local recurrence and long-term natural course of breast cancer, the prediction and evaluation of these events is very difficult. Therefore, validation using ICC analysis may be the optimal method to evaluate individual APBI techniques.

Although our multicatheter technique was demonstrated to be a reasonable option in APBI according to ICC analysis, 1 IBTR was observed. Although this recurrence was regarded as IBTR, it involved a tumor growing at a distance of approximately $1 \mathrm{~cm}$ from the lumpectomy cavity and had already been observed on contrast-enhanced MRI before initial surgery. We should strive to select cases according to not only reported criteria such as age, tumor biology, tumor size but also unifocal disease using detailed imaging analysis.

In summary, this observational study using ICC analysis has demonstrated that multicatheter APBI offers the same rates of local control as WBI in Japanese breast cancer patients. However, this study was not randomized, was based on only a small number of participants, and covered only a short follow-up period. Additional research information from several institutions in Asia is thus required to confirm the validity and generalizability of these findings.

\section{Acknowledgments}

The authors would like to thank Enago (www.enago.jp) for the English language review.

\section{References}

Athas, W. F., Adams-Cameron, M., Hunt, W. C., Amir-Fazli, A., \& Key, C. R. (2000). Travel distance to radiation therapy and receipt of radiotherapy following breast-conserving surgery. $J$ Natl Cancer Inst, 92, 269-271. http://dx.doi.org/10.1093/jnci/92.3.269

Ballard-Barbash, R., Potosky, A. L., Harlan, L. C., Nayfield, S. G., \& Kessler, L. G. (1996). Factors associated with surgical and radiation therapy for early stage breast cancer in older women. $J$ Natl Cancer Inst, 88, 716-726. http://dx.doi.org/10.1093/jnci/88.11.716

Beitsch, P. D., Wilkinson, J. B., Vicini, F. A., Haffty, B., Fine, R., Whitworth, P., ... Lyden, M. (2012). Tumor bed control with balloon-based accelerated partial breast irradiation: incidence of true recurrences versus elsewhere failures in the American Society of Breast Surgery MammoSite(®) Registry Trial. Ann Surg Oncol, 19, 3165-3170. http://dx.doi.org/10.1245/s10434-012-2489-x

Clarke, M., Collins, R., Darby, S., Davies, C., Elphinstone, P., Evans, E., ... Wang, Y.; Early Breast Cancer Trialists' Collaborative Group (EBCTCG). (2005). Effects of radiotherapy and of differences in the extent of surgery for early breast cancer on local recurrence and 15-year survival: An overview of the randomised trials. Lancet, 366, 2087-2106. 
Common Terminology Criteria for Adverse Events (CTCAE) version 3.0. $\mathrm{http} / / /$ ctep.cancer.gov/protocolDevelopment/electronic_applications/docs/ctcaev3.pdf

Darby, S. C., Ewertz, M., McGale, P., Bennet, A. M., Blom-Goldman, U., Brønnum, D., ... Hall, P. (2013). Risk of ischemic heart disease in women after radiotherapy for breast cancer. $N$ Engl $J$ Med, 368, 987-998. http://dx.doi.org/10.1056/NEJMoa1209825

Early Breast Cancer Trialists' Collaborative Group (EBCTCG), Darby, S., McGale, P., Correa, C., Taylor, C., Arriagada, R., ... Peto, R. (2011). Effect of radiotherapy after breast-conserving surgery on 10-year recurrence and 15-year breast cancer death: meta-analysis of individual patient data for 10,801 women in 17 randomised trials. Lancet, 378, 1707-1716.

Farrow, D. C., Hunt, W. C., \& Samet, J. M. (1992). Geographic variation in the treatment of localized breast cancer. $N$ Engl J Med, 326, 1097-1101. http://dx.doi.org/10.1056/NEJM199204233261701

Fisher, B., Anderson, S., Bryant, J., Margolese, R. G., Deutsch, M., Fisher, E. R., ... Wolmark, N. (2002). Twenty-year follow-up of a randomized trial comparing total mastectomy, lumpectomy, and lumpectomy plus irradiation for the treatment of invasive breast cancer. $N$ Engl $J$ Med, 347, 1233-1241. http://dx.doi.org/10.1056/NEJMoa022152

Fisher, B., Bryant, J., Dignam, J. J., Wickerham, D. L., Mamounas, E. P., Fisher, E. R., ... Wolmark, N., for the National Surgical Adjuvant Breast and Bowel Project. (2002). Tamoxifen, radiation therapy, or both for prevention of ipsilateral breast tumor recurrence after lumpectomy in women with invasive breast cancers of one centimeter or less. J Clin Oncol, 20, 4141-4149. http://dx.doi.org/10.1200/JCO.2002.11.101

Fowble, B., Solin, L. J., Schultz, D. J., Rubenstein, J., \& Goodman, R. L. (1990). Breast recurrence following conservative surgery and radiation: patterns of failure, prognosis, and pathologic findings from mastectomy specimens with implications for treatment. Int $J$ Radiat Oncol Biol Phys, 19, 833-842. http://dx.doi.org/10.1016/0360-3016(90)90002-2

Fyles, A. W., McCready, D. R., Manchul, L. A., Trudeau, M. E., Merante, P., Pintilie, M., ... Olivotto,. I. A. (2004). Tamoxifen with or without breast irradiation in women 50 years of age or older with early breast cancer. $N$ Engl J Med, 351, 963-970. http://dx.doi.org/10.1056/NEJMoa040595

Gabos, Z., Thoms, J., Ghosh, S., Hanson, J., Deschênes, J., Sabri, S., \& Abdulkarim, B. (2010). The association between biological subtype and locoregional recurrence in newly diagnosed breast cancer. Breast Cancer Res Treat, 124, 187-94. http://dx.doi.org/10.1007/s10549-010-1135-1

Gage I., Recht A, Gelman R, Nixon, A. J., Silver, B., Bornstein, B. A., \& Harris, J. R. (1995). Long-term outcome following breast-conserving surgery and radiation therapy. Int $J$ Radiat Oncol Biol Phys, 33, 245-251. http://dx.doi.org/10.1016/0360-3016(95)02001-R

Giess, C. S., Keating, D. M., Osborne, M. P., \& Rosenblatt, R. (1999). Local tumor recurrence following breast-conservation therapy: correlation of histopathologic findings with detection method and mammographic findings. Radiology, 212, 829-835.

Grobmyer, S. R., Lightsey, J. L., Bryant, C. M., Shaw, C., Yeung, A., Bhandare, N., ... Copeland, E. M. 3rd. (2013). Low-Kilovoltage, Single-Dose Intraoperative Radiation Therapy for Breast Cancer: Results and Impact on a Multidisciplinary Breast Cancer Program. $J$ Am Coll Surg, 216, 617-623. http://dx.doi.org/10.1016/j.jamcollsurg.2012.12.038

Hattangadi-Gluth, J. A., Wo, J. Y., Nguyen, P. L., Abi Raad, R. F., Sreedhara, M., Niemierko, A., ... Taghian, A. G. (2012). Basal subtype of invasive breast cancer is associated with a higher risk of true recurrence after conventional breast-conserving therapy. Int $J$ Radiat Oncol Biol Phys, 82, 1185-1191. http://dx.doi.org/10.1016/j.ijrobp.2011.02.061

Hughes, K. S., Schnaper, L. A., Berry, D., Cirrincione, C., McCormick, B., Shank, B., Norton, L., for the Cancer and Leukemia Group B, Radiation Therapy Oncology Group, and Eastern Cooperative Oncology Group (2004). Lumpectomy plus tamoxifen with or without irradiation in women 70 years of age or older with early breast cancer. $N$ Engl J Med, 351, 971-9777. http://dx.doi.org/10.1056/NEJMoa040587

Krauss, D. J., Kestin, L. L., Mitchell, C., Martinez, A. A., \& Vicini, F. A. (2004). Changes in temporal patterns of local failure after breast-conserving therapy and their prognostic implications. Int J Radiat Oncol Biol Phys, 60, 731-740. http://dx.doi.org/10.1016/j.ijrobp.2004.04.010

Lazovich, D. A., White, E., Thomas, D. B., \& Moe, R. E. (1991). Underutilization of breast-conserving surgery 
and radiation therapy among women with stage I or II breast cancer. JAMA, 266, 3433-3438. http://dx.doi.org/10.1001/jama.266.24.3433

Mary, B. K. (2011). Dramatic increase in the use of implantable accelerated partial breast irradiation after breast-conserving surgery. CA Cancer J Clin, 61, 205-206. http://dx.doi.org/10.3322/caac.20122

McCormick, B. (2005). Partial-breast radiation for early staged breast cancers: hypothesis, existing data, and a planned phase III trial. $J$ Natl Compr Canc Netw, 3, 301-307.

Millar, E. K., Graham, P. H., O'Toole, S. A., McNeil, C. M., Browne, L., Morey, A. L., ... Sutherland, R. L. (2009). Prediction of local recurrence, distant metastases, and death after breast-conserving therapy in early-stage invasive breast cancer using a five-biomarker panel. J Clin Oncol, 27, 4701-4708. http://dx.doi.org/10.1200/JCO.2008.21.7075

Mitsumori, M., \& Hiraoka, M. (2008). Current status of accelerated partial breast irradiation. Breast Cancer, 15, 101-107. http://dx.doi.org/10.1007/s12282-007-0012-1

Njeh, C. F., Saunders, M. W., \& Langton, C. M. (2010). Accelerated Partial Breast Irradiation (APBI): A review of available techniques. Radiat Oncol, 5, 1748-1717. http://dx.doi.org/10.1186/1748-717X-5-90

Ott, O. J., Hildebrandt, G., Pötter, R., Hammer, J., Lotter, M., Resch, A., ... Strnad, V. (2007). Accelerated partial breast irradiation with multicatheter brachytherapy: Local control, side effects and cosmetic outcome for 274 patients. Results of the German-Austrian multi-centre trial. Radiother Oncol, 82, 281-286.

Polgár, C., Fodor, J., Major, T., Németh, G., Lövey, K., Orosz, Z., ... Kásler, M. (2007). Breast-conserving treatment with partial or whole-breast irradiation for low-risk invasive breast carcinoma--5-year results of a randomized trial. Int J Radiat Oncol Biol Phys, 69, 694-702. http://dx.doi.org/10.1016/j.ijrobp.2007.04.022

Polgár, C., Major, T., Fodor, J., Németh, G., Orosz, Z., Sulyok, Z., ... Kásler, M. (2004). High-dose-rate brachytherapy alone versus whole breast radiotherapy with or without tumor bed boost after breast-conserving surgery: Seven-year results of a comparative study. Int J Radiat Oncol Biol Phys, 60, 1173-1181. http://dx.doi.org/10.1016/j.ijrobp.2004.05.012

Polgár, C., Sulyok, Z., Fodor, J., Orosz, Z., Major, T., Takácsi-Nagy, Z., ... Németh, G. (2002). Sole brachytherapy of the tumor bed after conservative surgery for T1 breast cancer: Five-year results of a phase I-II study and initial findings of the of a randomized phase III trial. J Surg Oncol, 80, 121-128. http://dx.doi.org/10.1002/jso.10110

Presley, C. J., Soulos, P. R., Herrin, J., Roberts, K. B., Yu, J. B., Killelea, B., ... Gross, C. P. (2012). Patterns of use and short-term complications of breast brachytherapy in the national medicare population from 2008-2009. J Clin Oncol, 30, 4302-4307. http://dx.doi.org/10.1200/JCO.2012.43.5297

Recht, A., Silen, W., Schnitt, S. J., Connolly, J. L., Gelman, R. S., Rose, M. A., ... Harris, J. R. (1988). Time-course of local recurrence following conservative surgery and radiotherapy for early stage breast cancer. Int J Radiat Oncol Biol Phys, 15, 255-61. http://dx.doi.org/10.1016/S0360-3016(98)90002-5

Sanghani, M., Truong, P. T., Raad, R. A., Niemierko, A., Lesperance, M., Olivotto, I. A., ... Taghian, A. G. (2010). Validation of a web-based predictive nomogram for ipsilateral breast tumor recurrence after breast conserving therapy. J Clin Oncol, 28, 718-722. http://dx.doi.org/10.1200/JCO.2009.22.6662

Sato, K., Mizuno, Y., Kato, M., Shimo, T., Kubota, J., Takeda, N., ... Okawa, T. (2012). Intraoperative open-cavity implant for accelerated partial breast irradiation using high-dose rate multicatheter brachytherapy in Japanese breast cancer patients: A single-institution registry study. Journal of Cancer Therapy, 3, 822-830. http://dx.doi.org/10.4236/jct.2012.325104

Sato, K., Takayanagi, H., Mizuno, Y., et al. (2011). Open-cavity implant for accelerated partial breast irradiation (APBI) using HDR multicatheter brachytherapy. Rinsho Geka, 66, 1669-1672. (abstract in Japanese)

Schroen, A. T., Brenin, D. R., Kelly, M. D., Knaus, W. A., \& Slingluff Jr, C. L. (2005). Impact of patient distance to radiation therapy on mastectomy use in early-stage breast cancer patients. J Clin Oncol, 23, 7074-7080. http://dx.doi.org/10.1200/JCO.2005.06.032

Smith, T. E., Lee, D., Turner, B. C., Carter, D., \& Haffty, B. G. (2000). True recurrence vs. new primary ipsilateral breast tumor relapse: an analysis of clinical and pathologic differences and their implications in natural history, prognoses, and therapeutic management. Int J Radiat Oncol Biol Phys 48, 1281-1289. http://dx.doi.org/10.1016/S0360-3016(00)01378-X

Smith, G. L., Xu, Y., Buchholz, T. A., Giordano, S. H., Jiang, J., Shih, Y. C., \& Smith, B. D. (2012). Association 
between treatment with brachytherapy vs whole-breast irradiation and subsequent mastectomy, complications, and survival among older women with invasive breast cancer. JAMA, 307, 1827-1837. http://dx.doi.org/10.1001/jama.2012.3481

Smith, G. L., Xu, Y., Buchholz, T. A., Smith, B. D., Giordano, S. H., Haffty, B. G., ... Shih, Y. C. (2010). Brachytherapy for Accelerated Partial-Breast Irradiation: A Rapidly Emerging Technology in Breast Cancer Care. J Clin Oncol, 29, 157-165. http://dx.doi.org/10.1200/JCO.2009.27.0942

Vaidya, J. S., Baum, M., Tobias, J. S., Wenz, F., Massarut, S., Keshtgar, M., ... Joseph, D. (2011). Long-term results of targeted intraoperative radiotherapy (Targit) boost during breast-conserving surgery. Int J Radiat Oncol Biol Phys, 8, 1091-1097. http://dx.doi.org/10.1016/j.ijrobp.2010.07.1996

Vaidya, J. S., Joseph, D. J., Tobias, J. S., Bulsara, M., Wenz, F., Saunders, C., ... Baum, M. (2010). Targeted intraoperative radiotherapy versus whole breast radiotherapy for breast cancer (TARGIT-A trial): an international, prospective, randomised, non-inferiority phase 3 trial. Lancet, 376, 91-102. http://dx.doi.org/10.1016/S0140-6736(10)60837-9

Veronesi, U., Cascinelli, N., Mariani, L., Greco, M., Saccozzi, R., Luini, A., ... Marubini, E. (2002). Twenty-year follow-up of a randomized study comparing breast-conserving surgery with radical mastectomy for early breast cancer. $N$ Engl J Med, 347, 1227-1232. http://dx.doi.org/10.1056/NEJMoa020989

Veronesi, U., Orecchia, R., Luini, A., Galimberti, V., Zurrida, S., Intra, M., ... Maisonneuve, P. (2010). Intraoperative radiotherapy during breast conserving surgery: a study on 1,822 cases treated with electrons. Breast Cancer Res Treat, 124, 141-151. http://dx.doi.org/10.1007/s10549-010-1115-5

Vinh-Hung, V., \& Verschraegen, C. (2004). Breast-conserving surgery with or without radiotherapy: Pooled-analysis for risks of ipsilateral breast tumor recurrence and mortality. J Natl Cancer Inst, 96, 115-121. http://dx.doi.org/10.1093/jnci/djh013

Yeo, S. G., Kim, J., Kwak, G. H., Kim, J. Y., Park, K., Kim, E. S., \& Han, S. (2010). Accelerated partial breast irradiation using multicatheter brachytherapy for select early-stage breast cancer: local control and toxicity. Radiat Oncol, 5, 56. http://dx.doi.org/10.1186/1748-717X-5-56 